\title{
Females Had a Worse T-Score at Lumbar Region and Males Had a Worse T-Score at Femoral Region among Chronic Kidney Disease
}

\author{
Hilal Al Kalbani', Issa Al Salmi²* (1), Fatma Al Rahbi², Sumaiya Al Siyabi ${ }^{3}$, Suad Hannawi ${ }^{4}$ \\ ${ }^{1}$ Rheumatology Department, The Royal Hospital, Muscat, Oman \\ ${ }^{2}$ The Renal Medicine Department, The Royal Hospital, Muscat, Oman \\ ${ }^{3}$ Department of Nuclear Medicine, The Royal Hospital, Muscat, Oman \\ ${ }^{4}$ Department of Medicine, Ministry of Health and Prevention, Dubai, UAE \\ Email: hilalmusabah@gmail.com, ^isa@ausdoctors.net, samaoman85@hotmail.com, siyabihs@yahoo.com, \\ ssiyabi2006@gmail.com,suad1@ausdoctors.net
}

How to cite this paper: Al Kalbani, H., Al Salmi, I., Al Rahbi, F., Al Siyabi, S. and Hannawi, S. (2022) Females Had a Worse T-Score at Lumbar Region and Males Had a Worse T-Score at Femoral Region among Chronic Kidney Disease. Open Journal of Nephrology, 12, 60-74.

https://doi.org/10.4236/ojneph.2022.121006

Received: January 17, 2022

Accepted: February 21, 2022

Published: February 24, 2022

Copyright $\odot 2022$ by author(s) and Scientific Research Publishing Inc. This work is licensed under the Creative Commons Attribution-NonCommercial International License (CC BY-NC 4.0). http://creativecommons.org/licenses/by-nc/4.0/ (c) (i) (2) Open Access

\begin{abstract}
Background: To evaluate bone-mineral-density-BMD determined by dualenergy X-ray absorptiometry-DEXA and bone turnover markers in chronic-kidney-disease-CKD patients. Method: An observational-clinical study of all patients who were scanned by DEXA-scan in 2018. All patients with lowbone-density or osteoporosis-based on World-Health-Organization-WHO definition were included. Results: 505 patients with abnormal-BMD, 87.3\% were in early-stage CKD-stage I - II, 8.5\% were in CKD-stage III - V and $4.2 \%$ did not have renal tests. 95 (18.8\%) were male with a mean age of 57.0 years and 410 (81.2\%) were females with a mean age of 55.8 years. Patients of $\geq 65$ years had lower T-score than those who were younger than 65 years-old. Among CKD patients, those with late-CKD (stage III - V) had less BMD-measurements and lower T-score than those with early-CKD (stage I - II). A significant positive correlation exists between parathyroid hormone-PTH-level and the lower T-score. Female had a worse T-score at the lumbar-region whereas male had a worse $\mathrm{T}$-score at the femoral-region. There was no significant difference between males and females for the T-score at hip-region. Conclusion: We observed a distribution of abnormal BMD among different age, sex and CKD groups. Measurements of BMD by DEXA might be a useful test to diagnose osteoporosis in CKD patients. Femoral and total hip areas were more affected, however DEXA might not be able to detect osteoporosis in the lumbar area of CKD patients. T-scores are lower in patients with more severe CKD and lower in elderly patients. PTH level is associated proportionally to the degree of bone loss. Early intervention and proper management must be implemented early among CKD patients with multidisciplinary team approach
\end{abstract}


strategy. More studies are needed to determine if DEXA techniques are enough to distinguish the quantity of bone loss between different stages of CKD.

\section{Keywords}

Bone-Mineral-Density-BMD, Dual-Energy X-Ray Absorptiometry-DEXA, Chronic-Kidney-Disease-CKD, T-Score, Lumbar Region, Femoral Region, Gender

\section{Introduction}

Chronic kidney disease (CKD) is a well-established risk factor for loss of bone mineral density (BMD) [1] [2] [3] [4] [5]. Metabolic changes, such as secondary hyperparathyroidism, elevated phosphate levels, impaired synthesis of 1,25dihydroxyvitamin $\mathrm{D}$, persistent metabolic acidosis, affect bone mineralization and cause abnormal BMD in patients with chronic kidney disease [6] [7]. Bone loss may vary between different stages of CKD. Renal osteodystrophy is the traditional term for bone lesions in conjunction with CKD and is now considered a part of the CKD-BMD. Bone turnover and bone volume may both be classified as high, normal or low [8]. Bone mineralization may be categorized as normal or abnormal [8]. Osteoporosis is characterized basically by decreased bone mass and it begins to appear in the pre-dialysis period [9] [10].

The work group of the Kidney Disease: Improving Global Outcomes (KDIGO) recommended in 2006 the use of the term chronic kidney disease-mineral and bone disorder (CKD-MBD) to describe a systemic disorder that incorporates these abnormalities [11] [12]. CKD-MBD includes abnormalities of calcium, phosphorus parathyroid hormone (PTH), fibroblast growth factor 23 (FGF23), and vitamin D metabolism. It includes abnormalities as well in bone turnover, mineralization, volume linear growth, or strength and Extra-skeletal calcification. The term "renal osteodystrophy" is now exclusively used to define alterations in bone morphology associated with CKD based upon bone biopsy [13] [14] [15].

Dual-energy X-ray absorptiometry (DEXA) is a standard method to determine BMD loss in patients with healthy kidney function [13] [16] [17]. On the other hand there is controversy, whether DEXA scan can assess BMD loss in patients with CKD [13] [16] [17]. A study conducted by Nickolas et al. reported that bone loss in patients with CKD cannot be discriminated by DEXA scan [18]. However, researchers have recommended to combine DEXA with biochemical and bone turn-over markers to yield better results in osteoporosis diagnosis in patients with end-stage kidney disease (ESKD) [15].

It has been noted that various factors including serum calcium, phosphate, parathyroid hormone (PTH), bone specific alkaline phosphatase (serum AP) interplay in the turnover bone disease in patients with CKD [19]. 
PTH is elevated progressively when function of kidney deteriorates, consequently elevated PTH levels make cortical bone more prone to catabolic effects while anabolic effects take rule in trabecular bone [20] [21]. Hence, the net effect results are increased turnover and occurrence of thickened and irregular bone.

In Oman, a recent survey found that of those aged 40 years and over, one percent of 40,000 participants had a severe kidney dysfunction, 9\% had moderate kidney dysfunction and $29 \%$ had a mild kidney dysfunction [22] [23]. Hence, this study is investigating the BMD by DEXA among CKD patients with various serum biochemical markers.

\section{Methods}

This is a cross-sectional study in patients referred for DXA scanning during 2018. The paper aims at describing BMD and biochemical biomarkers according to stages of chronic kidney disease (CKD).

All patients referred to DEXA scan with low bone density or osteoporosisbased on World Health Organization (WHO) definition were included for further analysis but excluded pediatric population.

As so, five hundred and five patients were enrolled in the study.

The regions of interest (ROI) that we measured were the left proximal femur (neck and trochanter), total hip and lumbar spine.

The T-score and Z-score, which is usually calculated for young ages $<50$ years, were studied. Variables including age, gender, different biochemical markers including calcium, alkaline phosphatase,, parathyroid hormone, and vitamin D level were studied.

We divided parathyroid hormone according to the international reference (Roch method insert) to $>6.9 \mathrm{pmol} / \mathrm{L}$ as high and $\leq 6.9 \mathrm{pmol} / \mathrm{L}$ as normal.

Laboratory tests of serum creatinine and estimated glomerular filtrations (eGFR) rate, if reported, were taken for all included patients, then were classified to different renal stages based on the international guideline group Kidney Disease Improving Global Outcomes (KDIGO) where the eGFR is calculated by MDRD equation. The presence of either proteinuria or microscopic hematuria with eGFR $>90 \mathrm{ml} / \mathrm{min} / \mathrm{m}^{2}$ was used to define stage I CKD as per KDIGO guidelines. CKD stage II defined as eGFR $<90$ to $60 \mathrm{ml} / \mathrm{min} / \mathrm{m}^{2}$. CKD III is defined as eGFR $<60-30$ whereas CKD IV is $<30$ to $15 \mathrm{ml} / \mathrm{min} / \mathrm{m}^{2}$. CKD V is defined as eGFR $<15 \mathrm{ml} / \mathrm{min} / \mathrm{m}^{2}$. In addition to the history of malignancy, radiation and hormonal therapy that could influence bone metabolism, we took into consideration the history of taking glucocorticoid, calcium, and vitamin D supplements.

The study was approved by the local ethics committee of Royal hospital (RH) in Muscat, Oman. The RH has an excellent medical record system where data is computerized and had received a well-recognized international certificate of excellence for the electronic medical system called "Al Shifaa". All data including clinical, laboratory and radiological data are collected prospectively in the Al- 
Shifaa.

A GE Lunar DPX-NT bone densitometry device was used for detection of BMD. The sections for detection included the anteroposterior lumbar spine (LS) (L2-L3-L4), left proximal femur (neck and troch) and total hip.

Diagnosis of osteoporosis is established by event of a fragility fracture or WHO value of T-score of $<-2.5$ at the lumbar spine, the femoral neck and total hip scores for patients who had no history of fragility fractures [13] [17]. T-score was defined as the number of SDs a person's BMD lies below the mean BMD for a sex-matched young healthy population.

According to the WHO criteria, patients were categorized into normal BMD ( $\mathrm{T}$-score $\geq-1.0$ ), low bone density ( $\mathrm{T}$-score $=-1.0$ to -2.5 ) and osteoporosis (T-score $<-2.5)$.

$\mathrm{Z}$-score was used to represent the SD below the mean BMD value that was normalized for an age-matched and sex-matched healthy population.

Those with Z-score were included in the study if the Z-score was less or equal to -2.0 and it was considered as low bone density rather than osteoporosis unless there is history of fragility fracture. The coefficient of variation for the DEXA machine was $0.7 \%$ in the LS and $1.0 \%$ in the proximal femur.

CKD patients were divided into two categories: standard group-early stageCKD (stages 1 - 2) and late CKD (stages 3 - 5).

Age was categorized firstly into two categories: $\geq 65$ years or $<65$ years to discriminate between old age vs non old age as conventionally, "elderly" has been defined as a chronological age of 65 years old or older in most of the developed countries. In parallel: it was then categorized into two categories $\geq 50$ years or $<50$ years to discriminate between menopause vs non-menopause, as the universal experience in women's aging, is the culmination of some 50 years of reproductive aging. In addition, men older than 50 years might have low testosterone levels with functional abnormalities at multiple levels of the hypothalamic-pituitary-testicular axis leading to hypogonadism and subsequent osteoporosis.

\section{Statistical Analysis}

SPSS (Statistical Package for Social Sciences) V.9.0 software was used for the statistical analysis. Student's t test was performed for the comparison of data between the two groups. One-way analysis of variance test was performed for the comparison of parameters between groups. The relationship between parameters was evaluated by Pearson correlation analysis. $\mathrm{P}$ value of $<0.05$ was considered statistically significant. All results were expressed as the mean \pm SD.

\section{Results}

During the period of the study, there were 505 patients with abnormal BMD aged 55.8 (16.0). Of which, 95 (18.8\%) were males aged with mean (SD) of 57.0 (20.5) years and $410(81.2 \%)$ were females with mean (SD) age of $55.4(14.8)$ 
years.

Among all patients with abnormal BMD: there were 270 (53.5\%) patients with CKD stage I, 171 (33.86\%) patients in CKD stage II, 35 (6.9\%) patients in CKD stage III, and $8(1.58 \%)$ patients in CKD stage IV and V.

The mean estimated GFR for patients who were $<65$ years was 89.1 (12.4) $\mathrm{ml} / \mathrm{min} / \mathrm{m}^{2}$, while the mean eGFR for patients $\geq 65$ years was $73.5(21.0) \mathrm{ml} /$ $\mathrm{min} / \mathrm{m}^{2}(\mathrm{P}<0.01)$.

The mean eGFR of patients with low bone density patients was 82.2 (14.6) $\mathrm{ml} / \mathrm{min} / \mathrm{m}^{2}$, while the mean eGFR in osteoporotic patient was $78.8(18.8) \mathrm{ml} /$ $\mathrm{min} / \mathrm{m}^{2}(\mathrm{P}=0.039)$.

The mean ALP in low bone density patients was 95.9 (59.3) iU/L, while the mean ALP in osteoporotic patients was 93.5 (59.6) iU/L with no statistically significant difference between the two groups.

Females had a worse T-score at lumbar region whereas males had a worse $\mathrm{T}$ score compared to females at femoral region whereas there was no significant difference at total hip region, as shown in Table 1.

Patients of 65 years and older had more lower T-score than those who were younger than 65 years old in both femoral and total hip T-scores $(\mathrm{P}=0.001)$, as shown in Table 2.

Femoral T-score was worsening in patients who were 65 years or older in late CKD compared to early CKD at the same age category $(\mathrm{P}=0.034)$, as shown in Table 3.

Table 1. Shows the T-score for lumbar, femoral and hip among male and female CKD patients.

\begin{tabular}{cccc}
\hline T-score & Sex & Mean (SD) & P value \\
\hline \multirow{2}{*}{ lumbar } & Male & $-1.251(-1.43)$ & 0.001 \\
& Female & $-1.745(-1.04)$ & \\
\hline \multirow{2}{*}{ Femoral } & Male & $-1.585(-1.28)$ & 0.030 \\
& Female & $-1.305(-0.95)$ & 0.429 \\
\hline \multirow{2}{*}{ Total Hip } & Male & $-1.001(-1.24)$ & \\
& Female & $-0.892(-1.05)$ &
\end{tabular}

$\mathrm{P}<0.05$ is significant.

Table 2. Shows the bone mineral density of lumbar, femoral and hip in CKD patient ( $<50$ years compared to $\geq 50$ years). T-score at lumbar, femoral and total hip of patient $\leq 65$ years vs. $>65$ years old.

\begin{tabular}{|c|c|c|c|c|c|c|c|c|c|c|c|}
\hline \multirow{2}{*}{$\begin{array}{l}\text { BMD } \\
\mathrm{g} / \mathrm{cm}^{2}\end{array}$} & \multicolumn{2}{|c|}{$<50$} & \multicolumn{2}{|c|}{$\geq 50$} & \multirow{2}{*}{ P-value } & \multirow{2}{*}{ T-score } & \multicolumn{2}{|c|}{$<65$} & \multicolumn{2}{|c|}{$\geq 65$} & \multirow{2}{*}{$P$-value } \\
\hline & Mean & SD & Mean & SD & & & Mean & SD & Mean & SD & \\
\hline Lumbar & 0.851 & 0.123 & 0.90 & 0.139 & 0.000 & Lumbar & -1.660 & 1.021 & -1.856 & 1.020 & 0.097 \\
\hline Femoral & 0.862 & 0.116 & 0.871 & 0.116 & 0.090 & Femoral & -1.120 & 0.992 & -1.849 & 0.909 & 0.000 \\
\hline Total Hip & 0.827 & 0.128 & 0.863 & 0.114 & 0.059 & Hip & -0.694 & 1.023 & -1.369 & 0.082 & 0.000 \\
\hline
\end{tabular}

BMD, Bone mineral density. $\mathrm{P}<0.05$ is significant. 
Table 3. Shows the T-score of early (stage I - II) CKD and the late (III - V) stage CKD among patients $<65$ years vs $\geq 65$ years.

\begin{tabular}{|c|c|c|c|c|c|c|c|c|c|c|}
\hline \multirow{3}{*}{ T-score } & \multicolumn{4}{|c|}{$<60$} & \multirow{3}{*}{$\mathrm{P}$-value } & \multicolumn{4}{|c|}{$\geq 65$} & \multirow{3}{*}{ P-value } \\
\hline & \multicolumn{2}{|c|}{ Early CKD } & \multicolumn{2}{|c|}{ Late CKD } & & \multicolumn{2}{|c|}{ Early CKD } & \multicolumn{2}{|c|}{ Late CKD } & \\
\hline & Mean & SD & Mean & SD & & Mean & SD & Mean & SD & \\
\hline Lumbar & -1.658 & 1.026 & -1.767 & 1.084 & 0.087 & -1.757 & 1.272 & -1.792 & 1.486 & 0.091 \\
\hline Femoral & -1.070 & 0.699 & -1.633 & 1.233 & 0.056 & -1.763 & 0.947 & -2.196 & 0.739 & 0.034 \\
\hline Total Hip & -0.691 & 1.021 & -0.785 & 1.206 & 0.074 & -1.334 & 1.103 & -1.648 & 0.994 & 0.104 \\
\hline
\end{tabular}

CKD, chronic kidney disease, $\mathrm{P}<0.05$ is significant.

Femoral BMD was lower in patients who were 50 years or older $(\mathrm{P}=0.100)$, however BMD lumbar measurements were higher in those who were also older than 50 years $(\mathrm{P}=0.000)$, as shown in Table 2 .

Patients with late CKD (stage III - V) had less BMD measurements and more lower T-score than those with early CKD (stage I - II) in both femoral and total hip, $\mathrm{P}$ values of 0.007 and 0.027 , as shown in Table 4 .

There was a trend towards statistical significance among Z-score between early (I - II stage) versus late (III - V) CKD, as shown in Table 5. The mean (SD) of T-score for lumbar, femoral and total hip among CKD patients with PTH $\leq 6.9$ $\mathrm{pmol} / \mathrm{L}$ versus $>6.9 \mathrm{pmol} / \mathrm{L}$, there were a significant positive correlation between PTH level and the lower T-score in both femoral and total hip, P values 0.003 and 0.008 , respectively. There was a trend towards a statistically significant negative relationship between PTH level and the lumbar T-score, as shown in Table 6.

\section{Discussion}

This study evaluated BMD among CKD patients who underwent DEXA scan. The population were of variable ages, majority were female, in early stage of CKD with eGFR of $82.2(14.6) \mathrm{ml} / \mathrm{min} / \mathrm{m}^{2}$ among patients with low bone density patients and $78.8(18.8) \mathrm{ml} / \mathrm{min} / \mathrm{m}^{2}$ in osteoporotic patients. Females had a worse $\mathrm{T}$-score at lumbar region whereas males had a worse $\mathrm{T}$-score compared to females at femoral region.

Also, this study showed that patients of 65 years and older had more lower T-score than those who were younger than 65 years old in both femoral and total hip T-scores. Femoral T-score was worsening in patients who were 65 years or older in late CKD compared to early CKD at the same age category.

In addition, the study showed that late CKD (stage III - V) had less BMD measurements and lower T-score than those with early CKD (stage I - II) in both femoral and total hip. There was a significant positive correlation between PTH level and the lower T-score in both femoral and total hip but non-significant negative relationship between PTH level and the lumbar T-score.

Hence, early management at early stages is of paramount importance even at early stages of CKD and at a younger age [24]. Osteoporosis is a metabolic bone 
Table 4. Shows the bone mineral density (BMD) and T-score of early (CKD I - II) versus late (III - V) stage CKD.

\begin{tabular}{|c|c|c|c|c|c|c|c|c|c|c|c|}
\hline \multirow{2}{*}{$\begin{array}{l}\text { BMD } \\
\mathrm{g} / \mathrm{cm}^{2}\end{array}$} & \multicolumn{2}{|c|}{ Early CKD } & \multicolumn{2}{|c|}{ Late CKD } & \multirow{2}{*}{ P-value } & \multirow{2}{*}{ T-score } & \multicolumn{2}{|c|}{ Early CKD } & \multicolumn{2}{|c|}{ Late CKD } & \multirow{2}{*}{$P$-value } \\
\hline & Mean & SD & Mean & SD & & & Mean & SD & Mean & SD & \\
\hline Lumbar & 0.885 & 0.133 & 0.932 & 0.147 & 0.034 & Lumbar & -1.688 & 1.105 & -1.343 & 1.331 & 0.070 \\
\hline Femoral & 0.782 & 0.116 & 0.731 & 0.112 & 0.007 & Femoral & -1.273 & 1.010 & -1.948 & 0.994 & 0.000 \\
\hline Total Hip & 0.838 & 0.133 & 0.789 & 0.188 & 0.027 & Hip & -0.879 & 1.084 & -1.238 & 1.179 & 0.050 \\
\hline
\end{tabular}

$\mathrm{BMD}$, Bone mineral density. CKD, chronic kidney disease. $\mathrm{P}<0.05$ is significant.

Table 5. Shows the Pearson correlation coefficient of the Z-score and CKD stages (95\% Confidence Interval of the Difference).

\begin{tabular}{ccccc}
\hline Z-score & Renal Stage & Mean score & SD & P value \\
\hline \multirow{2}{*}{ lumbar } & Early CKD & -1.554 & 4.984 & \multirow{2}{*}{0.079} \\
& Late CKD & -1.679 & 1.362 & \\
\hline \multirow{2}{*}{ Femoral } & Early CKD & -0.754 & 0.979 & 0.066 \\
& Late CKD & -0.824 & 1.213 & \\
\hline \multirow{2}{*}{ Total Hip } & Early CKD & -0.635 & 1.048 & \multirow{2}{*}{ Late CKD } \\
& -0.656 & 1.408 & \\
\hline
\end{tabular}

CKD, chronic kidney disease. $\mathrm{P}<0.05$ is significant.

Table 6. Shows the T-score for lumbar, femoral and hip among patients with PTH $\leq 6.9$ versus $>6.9 \mathrm{pmol} / \mathrm{L}$.

\begin{tabular}{ccccc}
\hline T-score & PTH & Mean & SD & P value \\
\hline \multirow{2}{*}{ lumbar } & $\leq 6.9$ & -1.000 & 0.707 & 0.072 \\
& $>6.9$ & -1.331 & 0.814 & \\
\hline \multirow{2}{*}{ Femoral } & $\leq 6.9$ & 0.000 & 1.414 & 0.003 \\
& $>6.9$ & -2.228 & 0.826 & 0.008 \\
\hline \multirow{2}{*}{ Total Hip } & $\leq 6.9$ & 1.110 & 1.131 & \\
\hline
\end{tabular}

$\mathrm{PTH}$, parathyroid hormone. $\mathrm{P}<0.05$ is significant.

disease of wide spectrum in CKD. Kanis JA had evaluated the "arguments that osteoporosis is in part due to either a defective 1 alpha-hydroxylase occurring at menopause or in later life or to target tissue resistance to calcitriol; some argue that disturbances in vitamin D metabolism are irrelevant to osteoporosis, being either a consequence of aging or a result, not a cause, of osteoporosis itself" [25]. Since vitamin D compounds can be targeted relatively late in the natural history of bone loss, and the effects on hip fracture appear to be marked, the use of vitamin D or alfacalcidol has a favorable cost-effectiveness ratio, which is increased markedly by the use of screening techniques to target interventions. However, E. Michael Lewiecki has argued that "more data are needed to better define the bone disease in patients with end-stage CKD, with or without di- 
abetes, and determine whether pharmacological interventions can reduce fracture risk with a favorable balance of benefit and risk" [26].

Abrahamsen et al. argued that managing osteoporosis, given reduced kidney function, is a major challenge in the bone clinic [27]. They, as well, concluded that additional data is crucial on the effectiveness and safety of bisphosphonates in the late stages-CKD and clinicians should reevaluate the low use of DXA in patients with the loss of kidney function in view of the existence of various options of managements availability [27]. Also, the high prevalence of secondary hyperparathyroidism and the high incidence of moderate impairment of kidney function suggests that more widespread use of these agents could make a substantial impact in the treatment of osteoporosis in the elderly [25] [28]. Bernard Cortet, also, stated that the central justification for this contraindication is the scarcity of data about using bisphosphonates for this population, as many phase III trials have excluded patients with low GFR and kidney-function. "Because of the age-related decline in kidney function, it is not unusual for elderly patients to have GFR ( $\mathrm{CrCl})$ below these cut-offs" [29].

The Bone strength is based on bone quantity and bone quality. The quality depends on physical composition, architecture, turnover, damage accumulation and mineralization whereas the bone density is determined by peak bone mass and amount of bone loss. Bone fracture risk is increased with osteoporosis. BMD as a predictor of fracture risk in non-renal population, CKD II and III is good. However, it is a moderate predictor for risk in CKD IV and V but quite variable among dialysis population.

Bone fracture risk is increased with osteoporosis. DEXA is the most widely used method in measuring BMD [17]. It is short scan time, cost-effective, and carries low radiation dose. Previous studies showed that DEXA gives good bone mass quantification and it enables measuring BMD in CKD patients. Others showed that DEXA obtaining BMD measurements is able to predict bone loss and risk of fracture in CKD patients, whether bone mass measurement can assess fracture risk in patients with CKD is still controversial [17]. This is because of different complex etiology as patients with CKD suffer from fractures due to impairments in bone quantity, bone quality, bone turnover markers and abnormalities of neuromuscular function particularly in stage IV and V.

A new term has emerged called CKD-MBD (mineral and bone disorder), where PTH and ALP increased, bone loss more at cortical bone, bone formation will be either very low in adynamic bone disease or sometimes very high, and it is strongly associated with vascular calcification [6] [11] [30]. All of this cause BMD to be weakly associated with fracture risk in patients with advanced CKD [14] [25]. Moreover, renal osteodystrophy can interfere with diagnosis of osteoporosis. Nevertheless, this concept is not yet conclusive. In our findings it was clearly shown that patients with late CKD i.e. stage IV and V had worsening BMD measurements.

$\mathrm{CKD}$ has been found to be associated with osteoporotic changes and fracture risk, which are increasing proportionally with the severity of the CKD and with 
the associated morbidity and mortality [13]. The duration of impaired kidney function could be considered as a risk factor for low BMD. The association between BMD obtained by DEXA and the severity of impaired kidney function have been investigated. Researchers found that femur and spine BMD is decreased as chronic kidney disease stages advanced [31] [32]. Other studies demonstrated the association between the loss of BMD and the eGFR deterioration [32].

Asian patients were studied by researchers, who reported that BMD of lumbar and femoral neck was positively associated with degree of CKD III and IV [26]. Similar findings were reported by others, who reported an association between abnormal BMD and kidney function impairment. Some studies showed that BMD is also lower in pre-dialysis as well as dialysis-dependent patients who fracture compared to those who do not [26] [28].

Our study demonstrated a significant relationship between lower BMD in the femur and total hip and the degree of CKD. This is in contrary to lumbar BMD which showed negative correlation with degree of CKD. The explanation of this is that DEXA cannot distinguish between cortical and cancellous bone, and it cannot assess bone microarchitecture or bone turnover as compared to the Magnetic resonance [33] [34].

A review of the literature in patients with CKD stage $V$ is suggesting the same as well and shows that bone density in the cortical bone of the hip is generally lower compared with normal reference ranges by about one to two standard deviations, Whereas, in the spine bone (lumbar) density tends to be closer to the average expected values

In patients with advanced CKD, the bone density using DEXA measurements is lost primarily from the cortical bone (hip), and it may be increased in the cancellous bone (lumbar, spine) [13] [16] [17] [32] [35] [36]. In general, our findings corresponds to the concept that Bone density of CKD patients in the cortical bone of the hip is generally lower compared with the normal reference range, but the spine bone density (cancellous or trabecular bone) may be within the normal range or even increased [5] [13] [16] [17] [32] [35] [36]. Moreover, our findings showed that late CKD patients were more likely to have osteoporosis than early CKD patients and these were more noticeable in the BMD of femoral and hip. However, we did not compare each stage of CKD individually due to sample size.

Using the cut off age of 50 years which is usually used to obtain Z-score in young patients and in predicting bone loss in hypogonadism male patients [1] [37] [38], our study demonstrated that bone loss was more prominent after the age of 65 years than after age of 50 years. However, in both categories there was observed ongoing bone loss and noticeable lower BMD measurements with age advance. There was no correlation between Z-score and degree of CKD, which could be attributed to the fact that Z-score is meant for normal population who are below 50 years, i.e. it is not necessarily reflecting bone loss in patient with risk factors such as those with $\mathrm{CKD}$, donating T-score could be more reliable test 
in patient with CKD regardless of age [1] [37] [38].

Our study demonstrated that femoral and total hip BMD measurements showed more correlation with age than the lumbar BMD. This could be explained by the fact that the lumbar BMD is affected by age sclerotic changes which could underestimate the BMD findings when age advances [39] [40] [41]. Though an older patient with $\mathrm{CKD}$ and low BMD and/or a fragility fracture may have osteoporosis or another bone and mineral disorder related to CKD (eg, hyperparathyroidism, adynamic bone disease, osteomalacia), this study showed significant decline in bone mass and femoral T-score in CKD patients when the age of the patients had been counted. The mean T-score in patients who were older than 65 years with late CKD was -2.2 while it was -1.8 in patients who were older than 65 years but with normal kidney function or early CKD. Similar picture was showed in total hip T-score when age advances i.e. older than 65 years but with less extent than that seen in femoral T-score. It has been showed that BMD provides information on risk for fracture in older individuals with or without moderate CKD [42] [43].

Identifying fracture risk by monitoring laboratory biomarkers is still has clinical limitations. In patients with CKD, however, raised levels of some biomarkers might be utilized with CKD stages [11] [19] [44]. In present study once abnormal BMD has been identified, the mean Alkaline phosphatase was not corresponding with degree of bone loss among osteoporotic versus low bone mass patients. However, it is not studied in this research whether ALP is a predictor risk tool for diagnosis of osteoporosis or fracture risk. Also, it was not studied if elevated ALP was associated with osteoporosis in patients with CKD.

It was reported in previous studies that bone specific alkaline phosphatase (BSAP) is the most sensitive and specific marker of bone remodeling in patients with CKD [29]. Interestingly, parathyroid hormone is associated proportionally, with significant $\mathrm{P}$ value, with bone loss when $\mathrm{CKD}$ advances. It is found in our study that elevated PTH is a good biomarker for the degree of bone loss mainly in femoral and total hip. These findings correspond to similar results in some previous studies: one has reported that PTH and $25(\mathrm{OH})$-Vit D could provide early diagnose of osteoporosis in patients with CKD [1]. Also, it has been shown that secondary higher levels of serum PTH was associated with decreased BMD. Similarly, others reported that higher PTH levels were associated with increased risk of BMD loss [6] [10].

Curtis et al., found that men with "higher PTH levels had approximately a 2 -fold greater rate of BMD loss compared with men with lower PTH levels, ir-

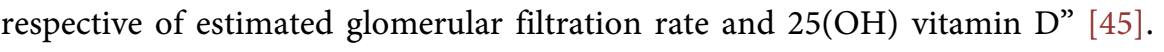
The ability of serum PTH to predict adynamic bone disease is predicated on the basis that the PTH synthesis is not being blunted by any pharmacologic agent (eg, vitamin D analogues, or cinacalcet). KDIGO (Kidney Disease Improving Global Outcomes) suggests using PTH trends rather than absolute targets to guide treatment decisions, although this strategy has not been tested specifically for distinguish osteoporosis from other elements of CKD-MBD, including renal 
osteodystrophy [11] [12].

\section{Limitations}

This is a single centre study. Also, this study did not examine whether DEXA scan can distinguish the quantity of bone loss between different stages of CKD. In addition, BSAP was not obtained in present study. Moreover, present study did not differentiate between pre-dialysis and dialysis patients in relation to BMD.

This study is lacking comparison between normal vs abnormal BMDs, as it included exclusively low bone density or osteoporotic patients only, i.e. we did not obtain similar data in a healthy control group. Regarding Z-score we need more research if it is useful for osteoporosis diagnosis in CKD patients in correspond to age and gender. Also, a single determination of BMD or T-scores, do not necessarily represent "bone loss". The BMD value could simply represent a low genetically pre-determined peak bone mass; however, this would be applicable to all the patients and not necessarily CKD people alone.

\section{Conclusions}

In this population, femoral BMD is lower at the hip in $\mathrm{CKD}$, and patients with $\mathrm{CKD}$ have lower bone loss than a reference population. Subsequently, it may demonstrate that in CKD patients, the impact of bone loss could be higher in femoral area than lumbar, and relatively both femoral and total hip areas are having more bone loss in CKD patients.

In general: BMD measurements obtained by DEXA and several biochemical markers of skeletal formation and resorption, PTH in our study, are useful in the diagnosis of bone status in CKD patients mainly in the femoral and hip areas. On the other hand, because of lack of information DEXA cannot distinguish bone loss quantity between different stages of CKD, which can restrict its use in these patient groups. There is a need for further studies on novel BMD measurement techniques and novel serum bone-turnover markers to determine bone loss quantity in patients with CKD.

\section{Compliance with Ethical Standards}

\section{Disclosure of Potential Conflicts of Interest}

The study was approved by the Scientific Research Committee at the Royal Hospital, Muscat, Oman, Ministry of health, and certify that the study was performed in accordance with the ethical standards as laid down in the 1964 Declaration of Helsinki and its later amendments ethical standards.

(https://mohcsr.gov.om/my-researches/)

\section{Informed Consent}

Each participant was freely given, informed consent to undergo procedure and laboratory investigations. 


\section{Availability of Data and Material}

Data of this paper is not available publicly but can be requested from the corresponding author in a reasonable time.

\section{Acknowledgements}

We would like to thank our patients and all the staff responsible for the delivery of patients' care. Also, we would like to thanks the following colleagues for their help in this study.

Fatma said khamis alhosni, Asma Mohammed Rashid Al Rubkhi, Asma Matar Al Maney, Jawahar Alshueili, Mustafa Ghaith Ahmed Thamer, Mustafa Al-Alawi, Lukman Salim Jamil Al Nomani, Intisar Saleh AL Malki.

\section{Financial Disclosure}

Hilal Al Kalbani, Issa Al Salmi, Fatma Al Rahbi, Sumaiya Al Siyabi, and Suad Hannawi declare that they have no conflict of interest.

\section{Conflicts of Interest}

The authors declare no conflicts of interest regarding the publication of this paper.

\section{References}

[1] Aggarwal, H.K., Jain, D., Yadav, S. and Kaverappa, V. (2013) Bone Mineral Density in Patients with Predialysis Chronic Kidney Disease. Renal Failure, 35, 1105-1111. https://doi.org/10.3109/0886022X.2013.815102

[2] Bezerra de Carvalho, K.S., Vasco, R.F.V., Custodio, M.R., Jorgetti, V., Moyses, R.M.A. and Elias, R.M. (2019) Chronic Kidney Disease Is Associated with Low BMD at the Hip but Not at the Spine. Osteoporosis International, 30, 1015-1023. https://doi.org/10.1007/s00198-019-04864-4

[3] Miller, P.D. (2014) Bone Disease in CKD: A Focus on Osteoporosis Diagnosis and Management. American Journal of Kidney Diseases, 64, 290-304.

[4] Miller, P.D. (2014) Chronic Kidney Disease and the Skeleton. Bone Research, 2, Article No. 14044. https://doi.org/10.1038/boneres.2014.44

[5] Pan, B.L. and Loke, S.S. (2018) Chronic Kidney Disease Associated with Decreased Bone Mineral Density, Uric Acid and Metabolic Syndrome. PLoS ONE, 13, e0190985. https://doi.org/10.1371/journal.pone.0190985

[6] Hou, Y.C., Lu, C.L. and Lu, K.C. (2018) Mineral Bone Disorders in Chronic Kidney Disease. Nephrology, 23, 88-94. https://doi.org/10.1111/nep.13457

[7] Iwasaki, Y., Kazama, J.J. and Fukagawa, M. (2017) Molecular Abnormalities Underlying Bone Fragility in Chronic Kidney Disease. BioMed Research International, 2017, Article ID: 3485785. https://doi.org/10.1155/2017/3485785

[8] McNerny, E.M.B. and Nickolas, T.L. (2017) Bone Quality in Chronic Kidney Disease: Definitions and Diagnostics. Current Osteoporosis Reports, 15, 207-213. https://doi.org/10.1007/s11914-017-0366-Z

[9] Bacchetta, J., Boutroy, S., Juillard, L., Vilayphiou, N., Guebre-Egziabher, F., Pelletier, S., et al. (2009) Bone Imaging and Chronic Kidney Disease: Will High-Resolution Pe- 
ripheral Tomography Improve Bone Evaluation and Therapeutic Management? Journal of Renal Nutrition, 19, 44-49.

[10] Carter, J.L., O’Riordan, S.E., Eaglestone, G.L., Delaney, M.P. and Lamb, E.J. (2008) Bone Mineral Metabolism and Its Relationship to Kidney Disease in a Residential Care Home Population: A Cross-Sectional Study. Nephrology Dialysis Transplantation, 23, 3554-3565. https://doi.org/10.1093/ndt/gfn302

[11] Ketteler, M., Block, G.A., Evenepoel, P., Fukagawa, M., Herzog, C.A., McCann, L., et al. (2017) Executive Summary of the 2017 KDIGO Chronic Kidney DiseaseMineral and Bone Disorder (CKD-MBD) Guideline Update: What's Changed and Why It Matters. Kidney International, 92, 26-36.

[12] Ketteler, M., Elder, G.J., Evenepoel, P., Ix, J.H., Jamal, S.A., Lafage-Proust, M.H., et al. (2015) Revisiting KDIGO Clinical Practice Guideline on Chronic Kidney Disease-Mineral and Bone Disorder: A Commentary from a Kidney Disease: Improving Global Outcomes Controversies Conference. Kidney International, 87, 502-528.

[13] Bover, J., Urena-Torres, P., Laiz Alonso, A.M., Torregrosa, J.V., Rodriguez-Garcia, M., Castro-Alonso, C., et al. (2019) Osteoporosis, Bone Mineral Density and CKDMBD (II): Therapeutic Implications. Nefrología, 39, 227-242. https://doi.org/10.1016/j.nefro.2018.10.009

[14] Damasiewicz, M.J. and Nickolas, T.L. (2018) Rethinking Bone Disease in Kidney Disease. JBMR Plus, 2, 309-322. https://doi.org/10.1002/jbm4.10117

[15] Khairallah, P. and Nickolas, T.L. (2018) Updates in CKD-Associated Osteoporosis. Current Osteoporosis Reports, 16, 712-723.

https://doi.org/10.1007/s11914-018-0491-3

[16] Bover, J., Urena-Torres, P., Torregrosa, J.V., Rodriguez-Garcia, M., Castro-Alonso, C., Gorriz, J.L., et al. (2018) Osteoporosis, Bone Mineral Density and CKD-MBD Complex (I): Diagnostic Considerations. Nefrología, 38, 476-490. https://doi.org/10.1016/j.nefro.2017.12.006

[17] Connelly, K., Collister, D. and Tangri, N. (2018) Fracture Risk and Treatment in Chronic Kidney Disease. Current Opinion in Nephrology and Hypertension, 27, 221 225. https://doi.org/10.1097/MNH.0000000000000411

[18] Nickolas, T.L. and Jamal, S.A. (2015) Bone Kidney Interactions. Reviews in Endocrine and Metabolic Disorders, 16, 157-163.

[19] Chiang, C. (2017) The Use of Bone Turnover Markers in Chronic Kidney DiseaseMineral and Bone Disorders. Nephrology, 22, 11-13.

https://doi.org/10.1111/nep.13014

[20] Robling, A.G., Kedlaya, R., Ellis, S.N., Childress, P.J., Bidwell, J.P., Bellido, T., et al. (2011) Anabolic and Catabolic Regimens of Human Parathyroid Hormone 1-34 Elicit Bone- and Envelope-Specific Attenuation of Skeletal Effects in Sost-Deficient Mice. Endocrinology, 152, 2963-2975. https://doi.org/10.1210/en.2011-0049

[21] Siddiqui, J.A., Johnson, J., Le Henaff, C., Bitel, C.L., Tamasi, J.A. and Partridge, N.C. (2017) Catabolic Effects of Human PTH (1-34) on Bone: Requirement of Monocyte Chemoattractant Protein-1 in Murine Model of Hyperparathyroidism. Scientific Reports, 7, Article No. 15300. https://doi.org/10.1038/s41598-017-15563-7

[22] Al Alawi, I.H., Al Salmi, I., Al Mawali, A. and Sayer, J.A. (2017) Kidney Disease in Oman: A View of the Current and Future Landscapes. Iranian Journal of Kidney Diseases, 11, 263-270.

[23] Al Ismaili, F., Al Salmi, I., Al Maimani, Y., Metry, A.M., Al Marhoobi, H., Hola, A., et al. (2017) Epidemiological Transition of End-Stage Kidney Disease in Oman. Kidney International Reports, 2, 27-35. 
[24] Kanis, J.A. (1995) The Use of Alfacalcidol in the Prevention of Bone Disease in Early renal Failure. Nephrology Dialysis Transplantation, 10, 23-28. https://doi.org/10.1093/ndt/10.supp4.23

[25] Kanis, J.A. (1999) Vitamin D Analogs: From Renal Bone Disease to Osteoporosis. Kidney International Supplements, 73, S77-S81.

[26] Lewiecki, E.M. (2012) Preventing Fractures in Diabetic Dialysis Patients. International Journal of Endocrinology and Metabolism, 10, 640-641.

[27] Abrahamsen, B., Ernst, M.T., Smith, C.D., Nybo, M., Rubin, K.H., Prieto-Alhambra, D., et al. (2020) The Association between Renal Function and BMD Response to Bisphosphonate Treatment: Real-World Cohort Study Using Linked National Registers. Bone, 137, Article ID: 115371. https://doi.org/10.1016/j.bone.2020.115371

[28] Kanis, J.A., McCloskey, E.V. and Beneton, M.N. (1997) Vitamin D and Analogues in Renal Bone Disease and Implications for Osteoporosis. Osteoporosis International, 7, 179-183. https://doi.org/10.1007/BF03194368

[29] Cortet, B. (2011) Osteoporosis Medications and Renal Failure. Joint Bone Spine, 78, 228-229. https://doi.org/10.1016/j.jbspin.2010.11.014

[30] Sugatani, T. (2018) Systemic Activation of Activin A Signaling Causes Chronic Kidney Disease-Mineral Bone Disorder. International Journal of Molecular Sciences, 19, Article No. 2490. https://doi.org/10.3390/ijms19092490

[31] Blomquist, G.A., Davenport, D.L., Mawad, H.W., Monier-Faugere, M.C. and Malluche, H.H. (2016) Diagnosis of Low Bone Mass in CKD-5D Patients. Clinical Nephrology, 85, 77-83. https://doi.org/10.5414/CN108708

[32] Fidan, N., Inci, A., Coban, M., Ulman, C. and Kursat, S. (2016) Bone Mineral Density and Biochemical Markers of Bone Metabolism in Predialysis Patients with Chronic Kidney Disease. Journal of Investigative Medicine, 64, 861-866.

[33] Leonard, M.B., Wehrli, F.W., Ziolkowski, S.L., Billig, E., Long, J., Nickolas, T.L., et al. (2019) A Multi-Imaging Modality Study of Bone Density, Bone Structure and the Muscle-Bone Unit in End-Stage renal Disease. Bone, 127, 271-279. https://doi.org/10.1016/j.bone.2019.05.022

[34] Sharma, A.K., Toussaint, N.D., Elder, G.J., Masterson, R., Holt, S.G., Robertson, P.L., et al. (2018) Magnetic Resonance Imaging Based Assessment of Bone Microstructure as a Non-Invasive Alternative to Histomorphometry in Patients with Chronic Kidney Disease. Bone, 114, 14-21. https://doi.org/10.1016/j.bone.2018.05.029

[35] Greenwood, C., Clement, J., Dicken, A., Evans, J.P., Lyburn, I., Martin, R.M., et al. (2016) Towards New Material Biomarkers for Fracture Risk. Bone, 93, 55-63. https://doi.org/10.1016/j.bone.2016.09.006

[36] Salas-Ramirez, M., Tran-Gia, J., Gbureck, U., Kosmala, A. and Lassmann, M. (2019) Quantification of the Trabecular Bone Volume Fraction for Bone Marrow Dosimetry in Molecular Radiotherapy by Using a Dual-Energy (SPECT/)CT. Physics in Medicine \& Biology, 64, Article ID: 205014. https://doi.org/10.1088/1361-6560/ab4476

[37] Jorgensen, H.S., Winther, S., Bottcher, M., Hauge, E.M., Rejnmark, L., Svensson, M., et al. (2017) Bone Turnover Markers Are Associated with Bone Density, but Not with Fracture in End Stage Kidney Disease: A Cross-Sectional Study. BMC Nephrology, 18, Article No. 284. https://doi.org/10.1186/s12882-017-0692-5

[38] Valkovsky, I., Olsanska, R., Tvrdik, J., Martinek, A., Svagera, Z., Pernicova, M., et al. (2015) Evaluation of Biochemical Markers and Bone Mineral Density in Patients with Chronic Kidney Disease Stage 5D at the Start of Hemodialysis Treatment. Biomedical Papers, 159, 93-99. 
[39] Miller, P.D. (2011) The Kidney and Bisphosphonates. Bone, 49, 77-81. https://doi.org/10.1016/j.bone.2010.12.024

[40] Shigematsu, T., Sonou, T., Ohya, M., Yokoyama, K., Yoshida, H., Yokoo, T., et al. (2017) Preventive Strategies for Vascular Calcification in Patients with Chronic Kidney Disease. Contributions to Nephrology, 189, 169-177. https://doi.org/10.1159/000452310

[41] Shindyapina, A.V., Mkrtchyan, G.V., Gneteeva, T., Buiucli, S., Tancowny, B., Kulka, M., et al. (2014) Mineralization of the Connective Tissue: A Complex Molecular Process Leading to Age-Related Loss of Function. Rejuvenation Research, 17, 116133. https://doi.org/10.1089/rej.2013.1475

[42] Chao, C.T., Yang, R.S., Huang, W.J., Tsai, K.S. and Chan, D.D. (2019) Risk Factors for Poor Functional Recovery, Mortality, Recurrent Fractures, and Falls Among Patients Participating in a Fracture Liaison Service Program. Journal of the American Medical Directors Association, 20, 1129-1136.e1.

[43] Yenchek, R.H., Ix, J.H., Shlipak, M.G., Bauer, D.C., Rianon, N.J., Kritchevsky, S.B., et al. (2012) Bone Mineral Density and Fracture Risk in Older Individuals with CKD. Clinical Journal of the American Society of Nephrology, 7, 1130-1136.

[44] Torres, P.A.U. and Cohen-Solal, M. (2017) Evaluation of Fracture Risk in Chronic Kidney Disease. Journal of Nephrology, 30, 653-661. https://doi.org/10.1007/s40620-017-0398-6

[45] Curtis, J.R., Ewing, S.K., Bauer, D.C., Cauley, J.A., Cawthon, P.M., Barrett-Connor, E., et al. (2012) Association of Intact Parathyroid Hormone Levels with Subsequent Hip BMD Loss: The Osteoporotic Fractures in Men (MrOS) Study. The Journal of Clinical Endocrinology \& Metabolism, 97, 1937-1944.

https://doi.org/10.1210/jc.2011-2431 